\title{
Design method of campus network structure robustness
}

\author{
Zhulin \\ Sichuan College of Architectural Technology, Library and Information Center, Deyang 618000, \\ China
}

Keywords: BP neural network; variable weight; robustness; network

\begin{abstract}
BP neural network to build the model for evaluating network information of network model, since the information exchange reflected in campus network is between individual and individual, randomness and model property factors of network information are prominent, BP neural network weights into curing condition, causes the information trust degree evaluation of large error. This paper presents an optimized robustness node design model, to construct information trust evaluation model. Setting up the trust degree respond weighting variable adaptive function periodically by adjusting network topologic weight vector, effectively reduce the operational cost of the iterative algorithm, and construct campus robust network. Simulation results show that, the new network information robustness evaluation model can reduce error rate of the website information evaluation greatly, the system has strong stability and robustness, can effectively promote the sound operation and development of network.
\end{abstract}

\section{Introduction}

In each big campus network, network trust crisis and network information security is ubiquitous [1], trust mathematical model modeling robustness of campus network information, stability and robustness analysis of network information are studied, to promote the healthy development of the campus network operation, which has great research value and practical value [2].

Traditionally, the robustness analysis and modeling study of campus network information is divided into two aspects of main object trust and subject trust. Blaze M. built the model for trust based on probability theory, and came up with the robustness analysis model of campus network structure and network user information and network information trust evaluation mechanism [3]; Olmedilla D. proposed the method to establish trust evaluation model by automatic consultation between the two sides [4]; Hu Wei, Jiang Zhengjun etc., utilized the neural network model to construct website model [5], has a certain role in improving stability and accuracy of network trust model, but unable to satisfy the exchange of information between the individual and the individual in campus network model, results in network information analysis weight need adaptive variation, robustness and model property factors of network information are prominent, causes the information trust degree evaluation of large error.

\section{2 evaluation system of campus network information robustness}

Under campus network mode, network information and evaluation system for trust and robustness, are the basis for determining the network operation and network information trust degree evaluation model provided by users, the campus network trust level evaluation index mainly includes: operators credibility, website credibility and exterior environment [6-8].

\section{Improvement of campus network robustness node topology design}

\subsection{The communication principle of campus network}

Based on the above setup of campus network model, this paper adopts the measure of uniform and random distribution mode, to process information capacity load distribution topology decomposition for node and edge vector of the network system model. During the normal operation of the whole network system, the total load is expressed as: 


$$
l\left(v_{v}\right)=l(a v)+l(c v)+l(b v)
$$

The load capacity of adjacent nodes $v_{a}, v_{b}$ and $v_{c}$ in campus network model are expressed as:

$$
\begin{gathered}
l\left(v_{a}\right)=l(b a)+l(c a) \\
l\left(v_{b}\right)=l(a b)+l(c b) \\
l\left(v_{c}\right)=l(a c)+l(b c)
\end{gathered}
$$

With the change of the campus network information, congestion is caused by growth of traffic, single vector equivalent weighted evaluation structure, the key channel and node in network cannot be effectively distinguished and evaluated, easy to cause the network structure destruction.

3.2 optimized model and key technologies

For the above problem, this paper proposes multi vector decomposition model. In the initial operation of network, if node $v_{v}$ is failed, before this, traffic load $l(v a), l(v b)$ and $l(v c)$ transmitted through node $v_{v}$ are not affected, however, nodes traffic flow $l(a v), l(b v)$ and $l(c v)$ which are not transmitted through the node will not transmit from this node, retrace to previous nodes $v_{a}, v_{b}$ and $v_{c}$, and recalculating the rout. At this time, traffic load of node $v_{a}, v_{b}$ and $v_{c}$ become:

$$
\begin{gathered}
l^{\prime}\left(v_{a}\right)=l(b a)+l(c a)+l(a v)=l\left(v_{a}\right)+l(a v) \\
l^{\prime}\left(v_{b}\right)=l(a b)+l(c b)+l(b v)=l\left(v_{b}\right)+l(b v) \\
l^{\prime}\left(v_{c}\right)=l(a c)+l(b c)+l(c v)=l\left(v_{c}\right)+l(c v)
\end{gathered}
$$

It can be seen from the above formula, when a catastrophic failure paralysis node ${ }^{v_{v}}$ appeared in campus network, the load is transferred to neighboring nodes $v_{a}, v_{b}$ and $v_{c}$ in a multi-layer vector state mode, at this time there will be:

$$
l^{\prime}\left(v_{i}\right)<c\left(v_{i}\right)(i=a, b, c)
$$

From the above analysis, it can be known that after uniform random decomposition is applied on the flow capacity of network destruction failure nodes, that is :

$$
\begin{aligned}
l\left(v_{v}\right) & =l(a v)+l(c v)+l(b v) \\
& =l(v a)+l(v c)+l(v b)
\end{aligned}
$$

The vector edge which connected to the node, that is channel information of connected nodes, these vector edge of channel information expressed as $e_{a v}, e_{b v}$ and $e_{c v}$, the loading capability of these edges are expressed as:

$$
\begin{aligned}
l\left(e_{a v}\right)=l(v a) & +l(a v) \\
l\left(e_{b v}\right) & =l(v b)+l(b v) \\
l\left(e_{c v}\right) & =l(v c)+l(c v)
\end{aligned}
$$

get:

$$
l\left(v_{v}\right)=\frac{1}{2}\left(l\left(e_{a v}\right)+l\left(e_{b v}\right)+l\left(e_{c v}\right)\right)
$$

Here, setting:

$$
l\left(e_{a v}\right)=l\left(e_{b v}\right)=l\left(e_{c v}\right)
$$

It has

$$
l\left(v_{v}\right)=\frac{3}{2} l\left(e_{a v}\right)
$$

Based on the previous formula, the node load is equal to half of the sum of adjacent edge load, namely network traffic capacity on the node is equivalent to half of total flow of its adjacent edges, distinguished evaluation is conducted for node model, for different nodes in the network, according to the relative location relationship of the center to process variable weight, node degree $d_{i}$ is defined, such as $d_{i}>2$, the relations of the capability of any failed node $v_{i}$ and average edge load capacity $\overline{l\left(e_{i}\right)}$ is :

$$
l\left(v_{i}\right)>\overline{l\left(e_{i}\right)}
$$


Wherein,

$$
\overline{l\left(e_{i}\right)}=\frac{\sum_{k} l\left(e_{i k}\right)}{d_{i}}
$$

Most of the node degree of entire network are greater than 2, which means that the nodes of campus network are more complex and less capacity, resulting in node crash and failure, therefore, in order to improve the survival ability of the network, there is the need to analyze the survivability and robustness of model in this paper.

\section{4 simulation experiments and results analysis}

According to the above new design method for the campus network robustness, combined with the campus network information trust degree evaluation standard, to process robustness investigation and simulation experiment, the simulation experiment is carried out in the Matlab simulation software, the research group obtained 12205 evaluation data from campus web site real-time software survey, from which 10000 data were selected as the training data, 2205 data were viewed as network system test data, the data was normalized, as shown in Figure 1. It can be seen from the figure that using this system to train the network, after 28 times training, can achieve the preset precision 0.001 .

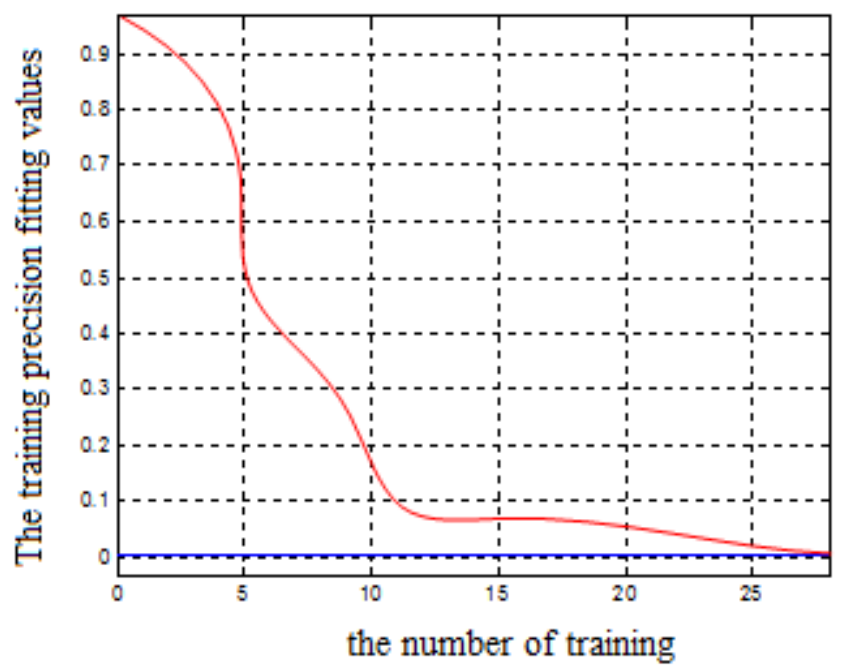

Figure 1 simulation results of BP neural network training times and training precision

Observed value and fitted value of expected output and the actual output of 8 groups of campus network information training samples were selected, 5 groups of test ample simulation were chosen, observation value and fitting value were shown in Table 1 and Table 2. It can be known from Table2, that the fitting value between expected output value and actual output value has small error, the system has better training effect, and strong robustness.

Table 1 observation value and the fitted value of the training sample

\begin{tabular}{ccccccccc}
\hline training sample & 1 & 2 & 3 & 4 & 5 & 6 & 7 & 8 \\
\hline observation value & 3.5 & 3 & 2.8 & 3.2 & 3 & 3 & 2.1 & 1.9 \\
the fitted value & 3.21 & 2.86 & 2.75 & 3.21 & 3.17 & 2.98 & 2.12 & 1.89 \\
\hline
\end{tabular}

Table 2 observation value and the fitted value of the test sample

\begin{tabular}{lccccc}
\hline \multicolumn{1}{c}{ test sample } & 1 & 2 & 3 & 4 & 5 \\
\hline observation value & 3.35 & 3 & 3.35 & 3 & 3 \\
the fitted value & 2.8635 & 2.8541 & 2.5487 & 3.2140 & 3.1256 \\
\hline
\end{tabular}

Training fitting value and training observed value output simulation of network samples topology training is as shown in Figure 2. It can be seen from Figure 2 that, through the optimized network topological structure system, fitting training used for training values only produce small error, which proves that the system for analyzing website network information have strong trust 
degree evaluation robustness, can withstand the adaptive control analysis under random exchange of personal information in campus network model, with high stability and good robustness.

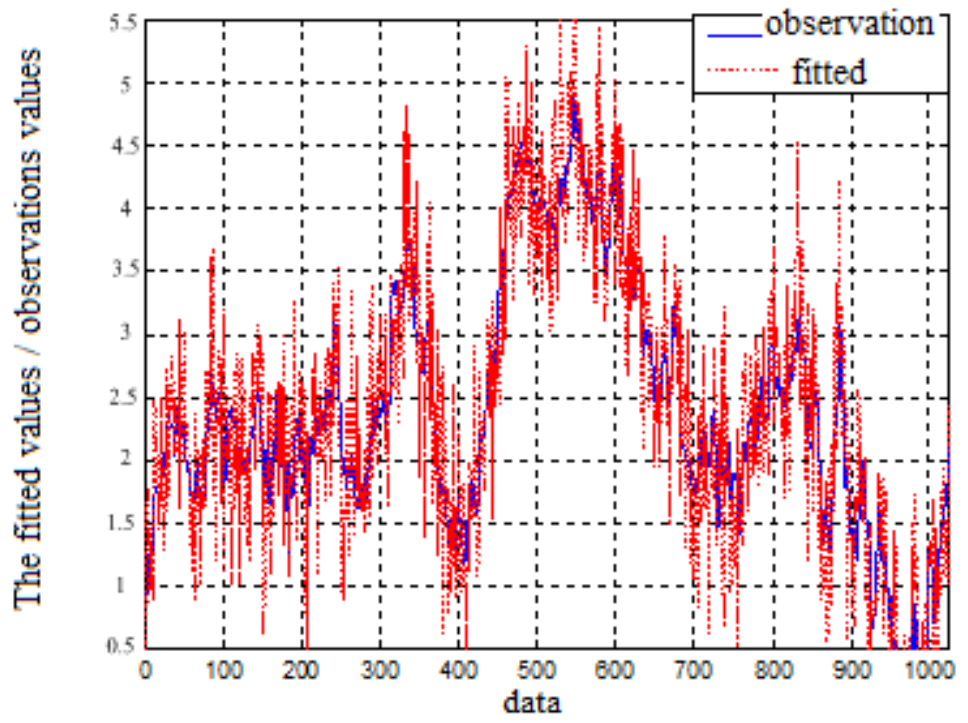

Figure 2 The output simulation of network sample topology training fitting value and training observed value

Based on the low error of fitting training network system experiment, the proposed campus network trust model was utilized to process the trust level training. From the results it can be seen that(Table 3 and table 4), output results and expected results were entirely consistent, the campus network information trust model system constructed by the proposed system is effective and reliable, achieve a better anti-interference.

Table 3 simulation results of the training sample's trust level

\begin{tabular}{ccccccccc}
\hline Training sample & 1 & 2 & 3 & 4 & 5 & 6 & 7 & 8 \\
\hline Expected & A & C & D & E & B & B & D & C \\
Actual & A & C & D & E & B & B & D & C \\
\hline
\end{tabular}

Table 4 the simulation results of the observation value and the output value of test sample

\begin{tabular}{cccccc}
\hline Test sample & 1 & 2 & 3 & 4 & 5 \\
\hline Expected & B & B & B & B & B \\
Actual & B & B & B & B & B \\
\hline
\end{tabular}

\section{Conclusion}

Study on the construction of trust evaluation mathematics model for network information and the robustness analysis. This paper presents an optimized robustness node design model, to construct information trust evaluation model. Setting up the trust degree respond weighting variable adaptive function periodically by adjusting network topologic weight vector, effectively reduce the operational cost of the iterative algorithm, and construct campus robust network. Simulation results show that, the new network information robustness evaluation model can reduce error rate of the website information evaluation greatly, the system has strong stability and robustness, can effectively promote the sound operation and development of network.

\section{References}

[1] Li Fei, Zhang Shibin. Study on the Network System Status's Assessment and Forecast Model Based on Cloud Model [J]. Journal of Sichuan University, 2010, 42 (6): 99-104.

[2] Ding Ling, Zhao Xiaogang. An improved BP neural network for intrusion detection [J]. Micro computer information, 2012, 28 (3): 131-134.

[3] Blaze M., Feigenbaum J., Lacy J. Decentralized trust management [J], Proceedings of the 
Symposium on Security and Privacy. Oakland, 1996: 164-173.

[4] Xi Rongrong, Yun Xiaochun, Jin Shuyuan, et al. Research survey of network security situation awareness [J]. Computer applications, 2012, 32 (1): 1-4.

[5] Hu Weixiong, ginger preserved. A Trust Evaluation Model for C2C E-Commerce Based on BP Neural Network [J]. Library and information work, 2012, 56 (10): 131-137.

[6] Wang Shouxin, Zhang Li, Li Hesong. Evaluation Approach of Subjective Trust Based on Cloud Model [J]. Journal of software, 2010, 21 (6): 1341-1352.

[7] Hou Limin, Zhang Ruikun. QoS Multicast Routing Algorithm Based on Agent and Simulation [J]. Computer simulation, 2011, 28 (1): 140-144.

[8] Zhang defined. Chemical Industrial Park Hazards Monitoring System Design Based on the Content of the Networking and ZigBee [J]. Bulletin of Science and Technology, 2012, 28 (8): 201- 204 